ESAIM: PROCEEDINGS, November 2002, Vol.12, 140-145

M.Thiriet, Editor

\title{
NUMERICAL SIMULATIONS OF BLOOD FLOWS IN ARTERIES FOR INTERVENTIONAL MEDICINES
}

\author{
Stéphanie Salmon ${ }^{1}$, Marc Thiriet $^{1}$ and Jean-Frédéric Gerbeau ${ }^{1}$
}

\begin{abstract}
We present and discuss some numerical simulations of blood flow in two realistic geometries of diseased vessels reconstructed from medical imaging. We prove by exhibiting valuable informations that these simulations can improve the planning of mini-invasive interventions used for treating some vascular diseases as stenoses or aneurisms.
\end{abstract}

Résumé. Dans ce papier, nous présentons des simulations numériques d'écoulement sanguin dans deux géométries de vaisseaux malades reconstruits à partir d'images médicales. A l'aide des informations obtenues, nous démontrons l'intérêt de ces simulations pour la planification des interventions mini-invasives pratiquées pour traiter ces maladies vasculaires.

\section{INTRODUCTION}

Modelization and numerical simulations of blood flows in aneurisms or stenoses, major vascular diseases, can play a new role as they can provide information on haemodynamical factors which contribute to the prognosis of these diseases. Moreover, it can help treatment planning and ensure better therapy. The joint investigation is aimed at developing simulators for interventional medicine based on numerical simulations of blood flows in diseased vessels meshed after three-dimensional reconstruction from medical imaging [1]. In particular, we want to provide a flexible computational support to plane the operation and predict complication risks by computing blood and vascular-wall stresses.

In the sequel, we present three-dimensional numerical simulations of blood flows in two different aneurisms. The first section is devoted to the flow modeling and the boundary conditions prescribed on the vessels. In Section 2, we discuss the results and present some informations.

\section{FLOW MODELING}

Blood, concentrated suspension of cells, is a non-newtonian fluid. In the present work, the time constant of cell agregation is supposed greater than the time scale. So we suppose that the blood is incompressible, homogeneous and newtonian and we work with arteries, the biggest vessels. The code is a 3D instationary Navier-Stokes finite element code developped at INRIA [2]. The numerical scheme is classsical : the pressure is linear and the velocity is discretized with linear functions plus a bubble function $\left(\mathbb{P}^{1}-\mathbb{P}^{1}+\right.$ bubble), associated to a characteristic method in time e.g [3]. The initial condition is given by a stationary Stokes problem with the same boundary condition as the unsteady one.

${ }^{1}$ INRIA Rocquencourt - ARC VITESV

B.P.105 F-78153 Le Chesnay Cedex

FRANCE

(c) EDP Sciences, SMAI 2003

Article published by EDP Sciences and available at http://www.edpsciences.org/proc or http://dx.doi.org/10.1051/proc:2002023 


\subsection{Inlet boundary conditions}

We prescribe a time-dependent uniform velocity at the inlet, which is derived from "in vivo" MR velocimetry. We apply the Fourier transform on the points obtained experimentally and obtain a time-dependent velocity (Fig. 1). Besides, velocity condition is chosen rather than pressure condition because of non-invasive measurements.

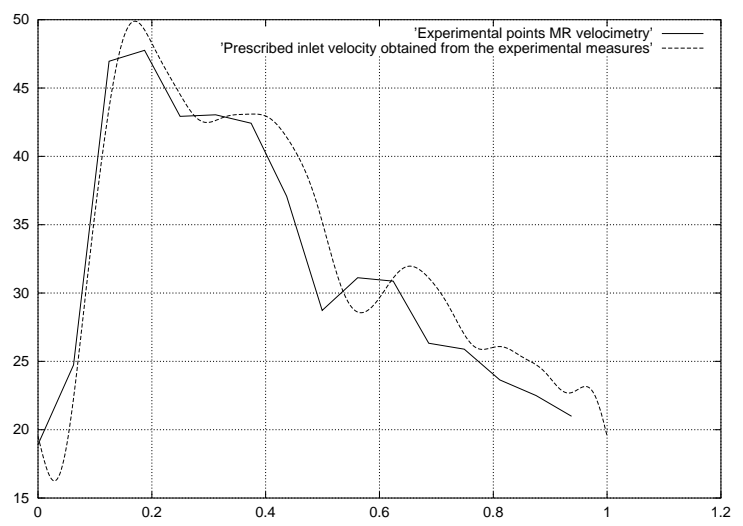

FigURE 1.Inlet boundary conditions - Velocity.

\subsection{Outlet boundary conditions}

The outlet sections are perpendicular to the axis and belong to straight pipes [1] in order to get uniform pressure and normal constraint equal to zero at exits.

\section{RESUlTS AND DISCUSSION}

Note that we have compared results on different periods to ensure that the phenomenon observed is reproductible. The very good correspondance between the periods is illustrated on Fig.2.
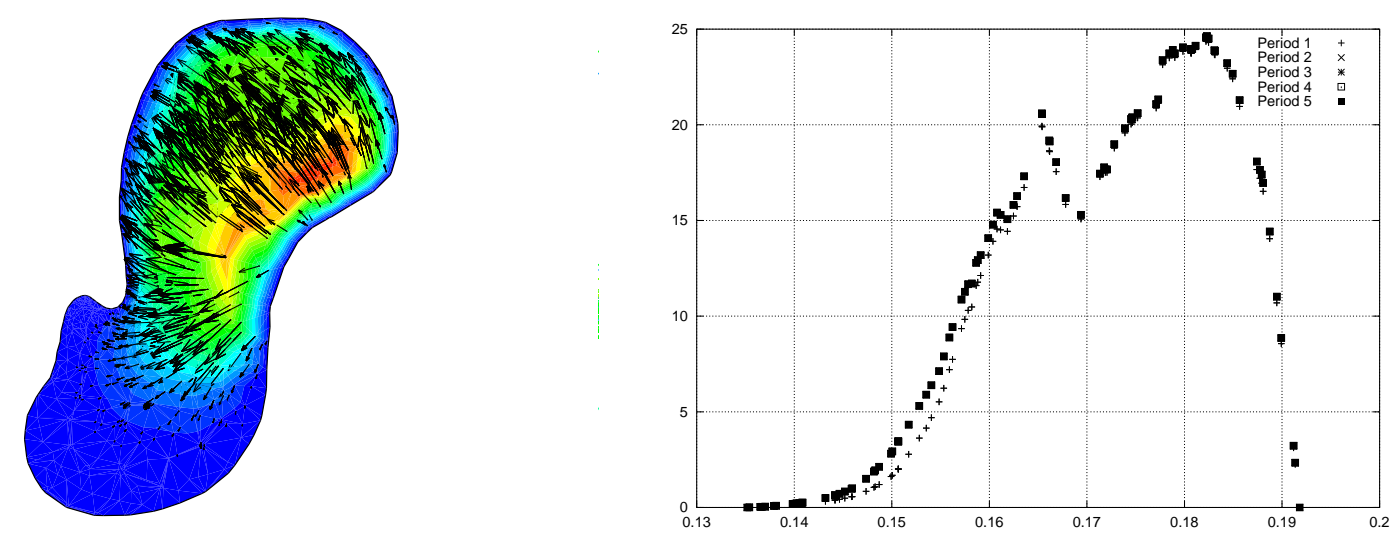

FIGURE 2.Velocity norm on the line (left) in a section in the cerebral congenital aneurism - Re $=310$ Comparison between 5 periods in the accelerating phase. 
On Fig. 3, 4 and 5, some different planes or same plane at different time on the cycle in both aneurisms are shown. Vectors or streamlines represent the velocity field and colors the pressure field. We can easily deduce from the computation that there is a risk of rupture where the pressure is high. The intervention to prevent the rupture consists in an implantation of a coil to fill in the aneurism and then stop the blood flow in the cavity. From the computation, we can plan where the coil should be implanted to minimize the risk.

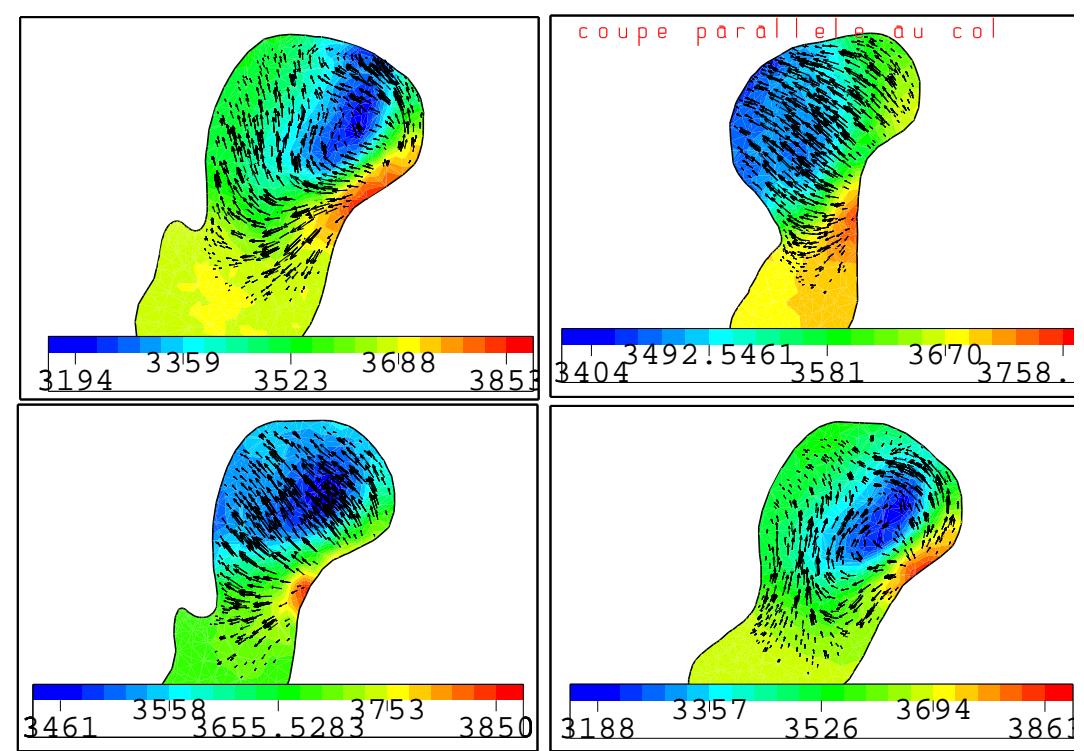

FIgURE 3.Pressure field and velocity vectors in different plane sections of the cerebral aneurism : top left, bottom left and right : sagital, top right : transversal.

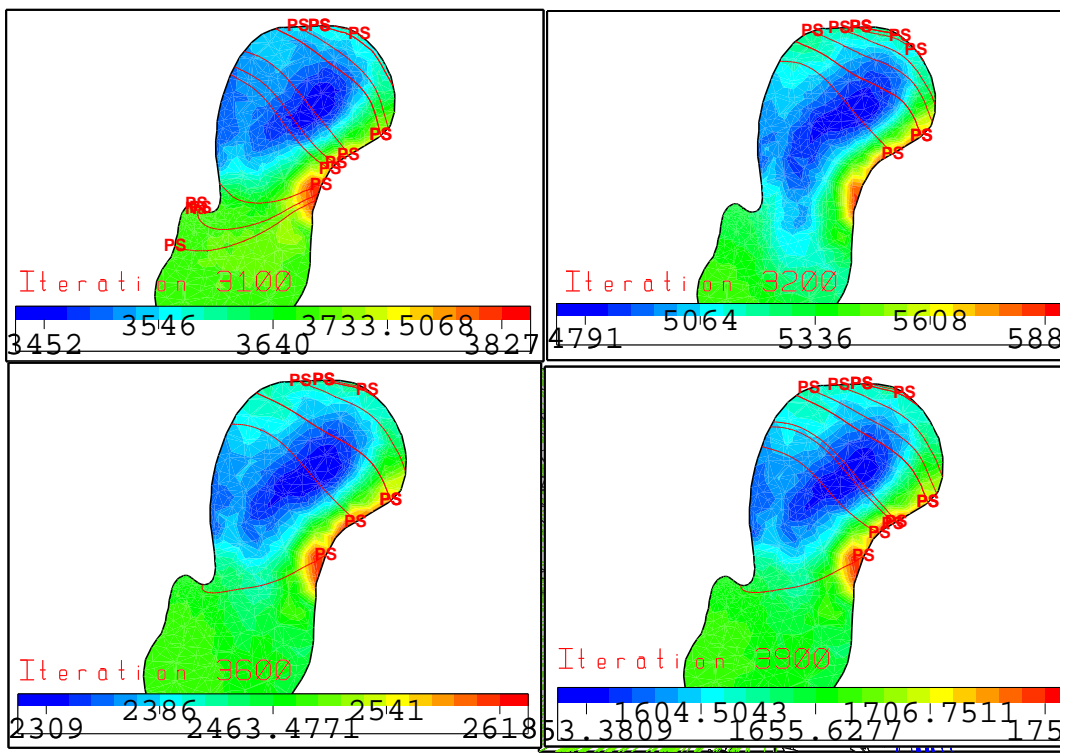

Figure 4.Pressure field and critical points in different plane sections of the cerebral aneurism - Different iterations in a period $(\mathrm{t}=0.1, \mathrm{t}=0.2, \mathrm{t}=0.6, \mathrm{t}=0.9)$. 

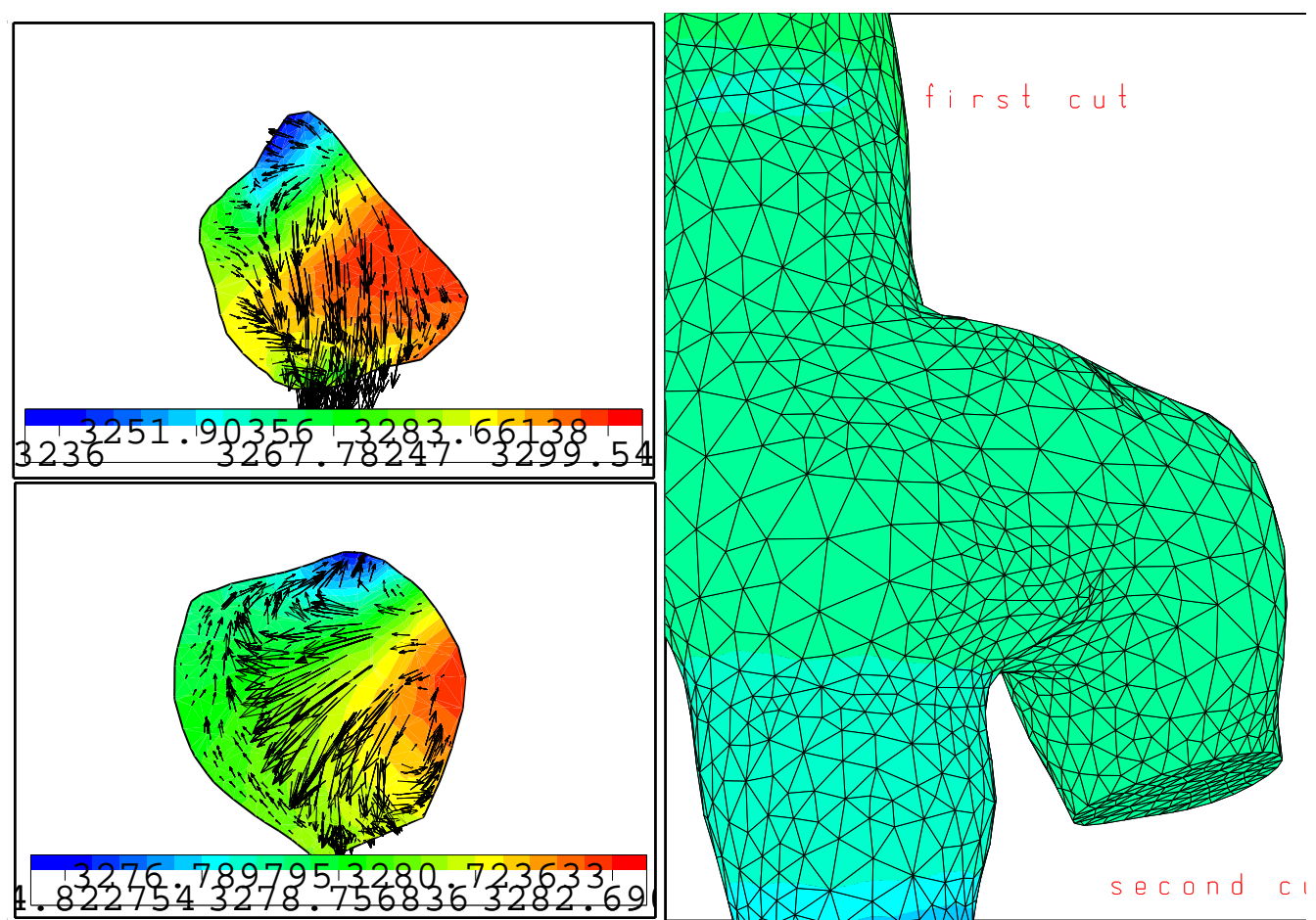

FiguRE 5.Pressure field and critical points in different plane sections of the iliac aneurism.

To conclude numerical results confirm what seems to be sensible : that an edge aneurism like the one on the iliac artery have much lower risk of rupture than a branching-site, either lateral or terminal, aneurism as the investigated cerebral one. Indeed, the flow is much more important in the cerebral aneurism than in an edge one (Fig. 6 and 7). Moreover, the normalized pressure is higher in the cerebral aneurism than in the iliac one (Fig. 8 and 9).

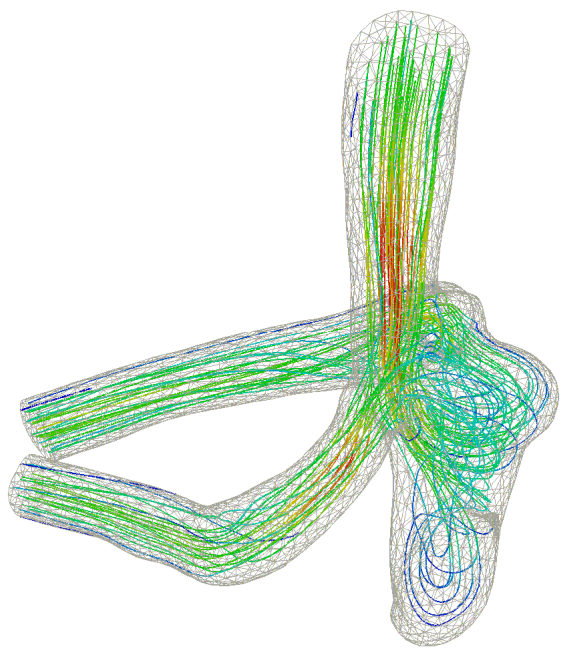

FiguRE 6.Streamlines in the cerebral aneurism - Re $=310$. 


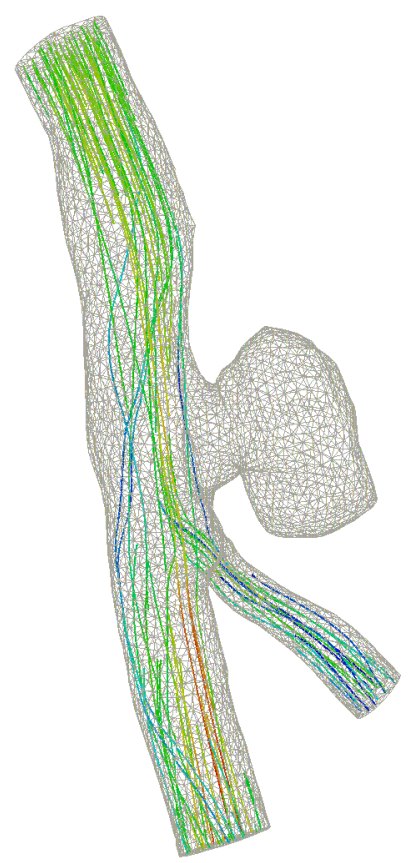

FIGURE 7.Streamlines in the iliac aneurism - Re $=750$.
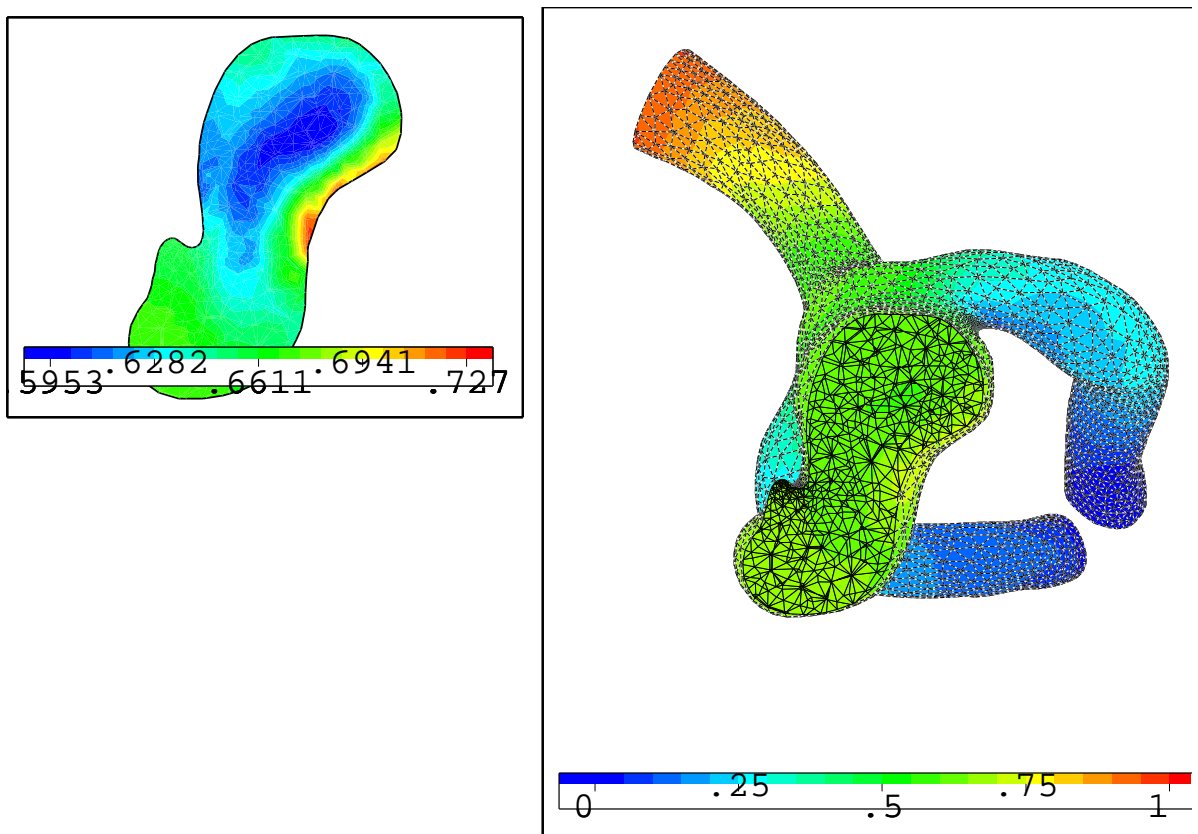

Figure 8.Normalized pressure in a plane (left) of the cerebral aneurism - Re $=310$ - Peak flow. 

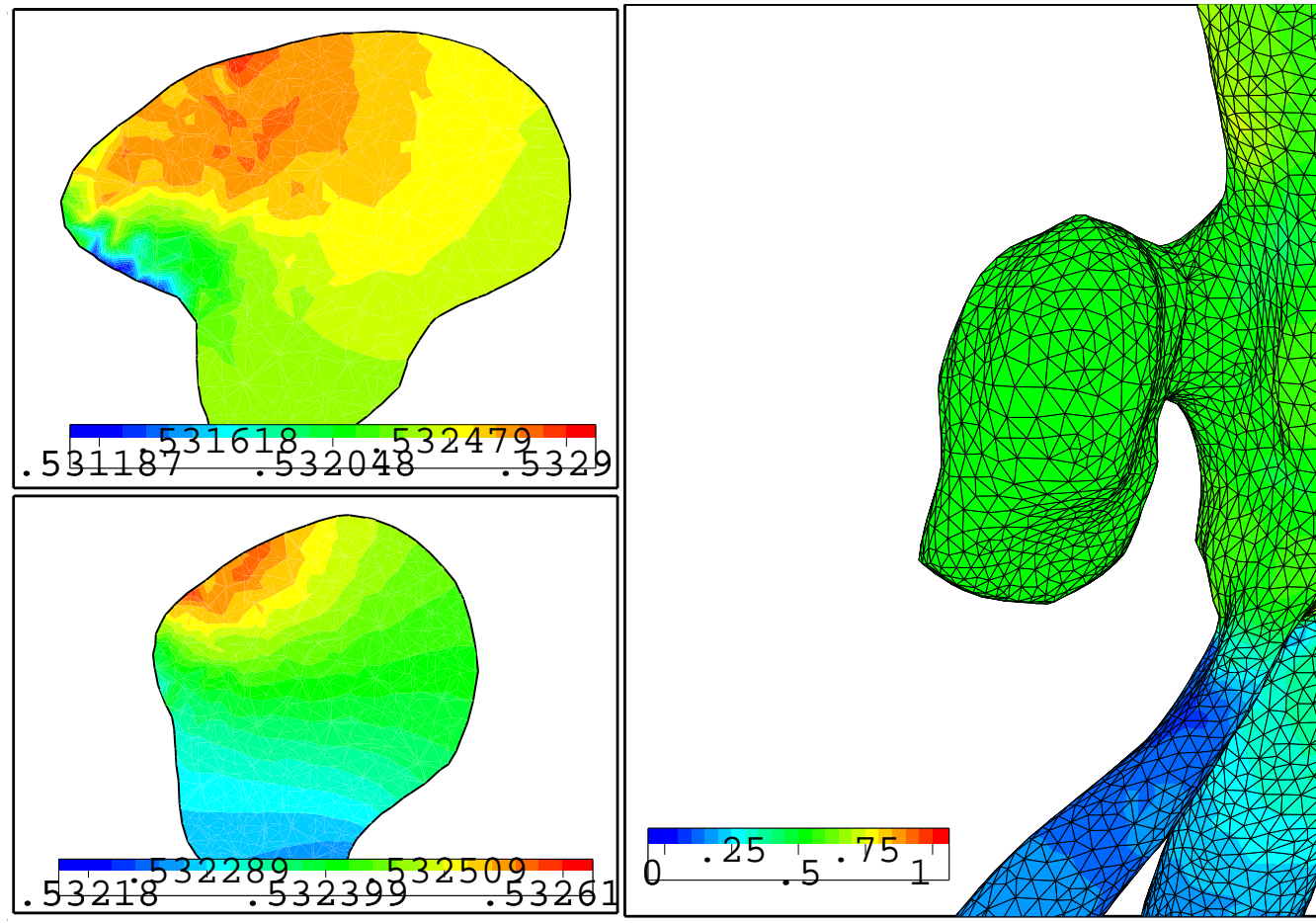

FiguRE 9.Normalized pressure in planes (left) of the iliac aneurism - Re $=750$ - Peak flow.

\section{REFERENCES}

[1] J.-D. Boissonat, P. Frey, G. Malandain, F. Nicoud, S. Salmon, E. Saltel, M. Thiriet, From medical images to computational meshes, Abstract MS4CMS02, 2002.

[2] F. Hecht, C. Parès, NSP1B3 : un logiciel pour résoudre les équations de Navier Stokes incompressible 3D, INRIA's Research Report 1449, 1991.

[3] V. Girault, P-A. Raviart, Finite element method for Navier-Stokes equations. Springer Verlag, Springer Series in Computational Mathematics, 1986. 